\title{
Motion of mitral apparatus in hypertrophic cardiomyopathy with obstruction
}

\author{
J. Christine Rodger \\ From Division of General Medicine, Stobhill General Hospital, Glasgow
}

Motion of the mitral apparatus in hypertrophic cardiomyopathy with obstruction was investigated by conventional single dimensional and multidimensional echocardiography. In systole, anterosuperior displacement of the posterior papillary muscle, failure of mitral valve closure, and anterior motion of both mitral leaflets were shown. The anterior leaflet was seen to impinge on the posterior papillary muscle but not on the interventricular septum in systole. The abnormality of the single dimensional mitral echogram, previously ascribed to systolic anterior motion of the mitral anterior leaflet, was found to be a complex of echoes from the chordae tendineae, the papillary muscle, and, furthest from the septum, the mitral anterior leaflet.

It is concluded that systolic anterior motion of the mitral anterior leaflet is of smaller amplitude than others have suggested, and that obstruction to left ventricular outflow in hypertrophic cardiomyopathy is produced by systolic contact between the mitral anterior cusp and the posterior papillary muscle. The theory is put forward that displacement of the posterior papillary muscle above and in front of the mitral leaflets produces chordal slackening, and that it is displacement of the chordae tendineae by the blood flowing to the aortic root during left ventricular ejection, which is responsible for systolic anterior motion of the mitral leaflets.

In patients who have hypertrophic cardiomyopathy with obstruction, systolic anterior movement of the mitral anterior cusp can be shown angiographically (Simon, 1968; Reis et al., 1974). The workers who described the systolic abnormality of the single dimensional mitral echogram in this disorder, attributed it to abrupt systolic anterior motion of the mitral anterior leaflet (Shah, Gramiak, and Kramer, 1969; Pridie and Oakley, 1970), and so far their conclusions have not been challenged (Tajik and Giuliani, 1974; Rossen et al., 1974; Bolton et al., 1974; Henry et al., 1975). Though possible mechanisms have been suggested (Shah et al., 1969; Pridie and Oakley, 1970; Reis et al., 1974; Henry et al., 1975), the cause of the abnormal anterior movement has not bean defined.

Single dimensional and multidimensional echocardiography (Roelandt et al., 1974) were used to investigate motion of the mitral apparatus in hypertrophic cardiomyopathy. The findings are presented in this paper: in the light of them, the systolic abnormality of the conventional echocardiogram is reinterpreted and a new explanation of systolic anterior motion of the mitral leaflets is proposed. Received 8 January 1976.

\section{Patients and methods}

Four patients, three of them male, were studied: their ages ranged from 12 to 58 years. The clinical diagnosis of hypertrophic cardiomyopathy with obstruction had been confirmed by cardiac catheterization and all had a left ventricular outflow gradient at rest. Two were on treatment with betaadrenergic blocking agents.

Single dimensional and, at a later date, multidimensional echocardiograms were recorded with the patients resting and semirezumbent. The single and the multiple element transducers were positioned to display maximal motion of the mitral anterior cusp and a long-axis view of the left ventricle, respectively.

The equipment for the single dimensional study was an Eskoline 20 ultrasonoscope (Smith Kline Instruments) interfaced with an ultraviolet strip chart recorder (Honeywell Visicorder). The multidimensional echocardiograms were recorded with a multiple element ultrasound system (Organon Teknika Echocardiovisor) which displays a moving two-dimensional echo image of the heart on an oscilloscope (Roelandt et al., 1974). The dynamic 


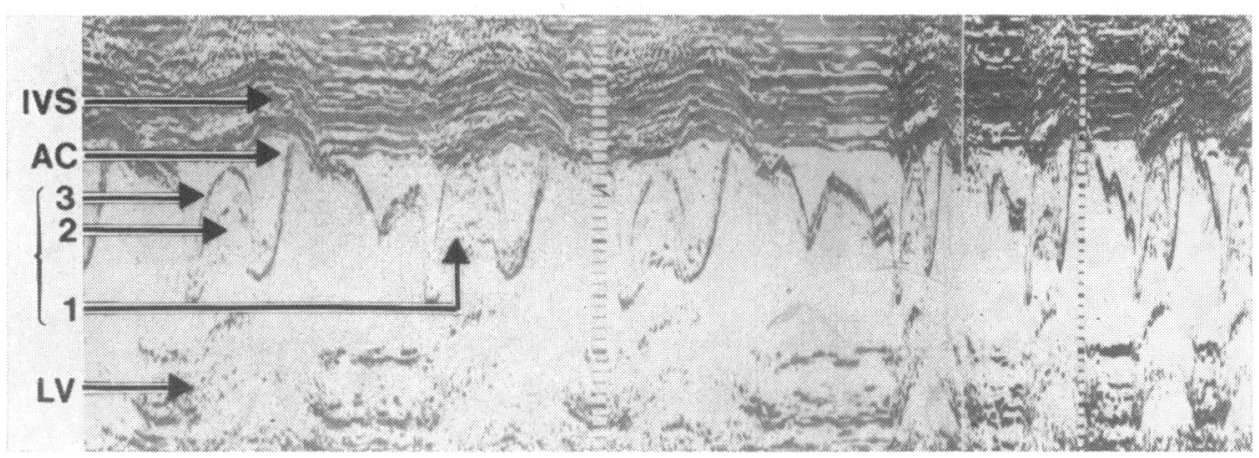

FI G. 1 Single dimensional echocardiogram from a patient with hypertrophic cardiomyopathy with obstruction and atrial fibrillation. The echoes forming the systolic complex (see text) are numbered. In this and subsequent figures: $A C=$ mitral anterior cusp, IVS=interventricular septum, $L V=$ left ventricular posterior wall.

display was stored on videotape using a slave oscilloscope and a television camera. Static Polaroid records were taken from the master oscilloscope with a camera triggered by the electrocardiogram (as in Fig. 3 and 5) and also from the videotape, using the stop-action facility on the video-replay system (as in Fig. 4 and 6).

\section{Results}

\section{Single element scans}

The mitral echogram comprised a single diastolic echo and multiple systolic echoes (Fig. 1).

Diastole A typical mitral anterior cusp echogram was recorded in diastole: the anterior cusp opened forwards impinging on the interventricular septum and closed at end-diastole to a point behind its endsystolic position.

Systole The echo complex recorded in systole had three components (Fig. 1, echoes 1, 2, and 3). First, a posterior echo (Fig. 1, echo 1), which was in continuity with the anterior cusp echo at enddiastole and end-systole and which moved anteriorly to a mid-systolic position well behind the interventricular septum. Second, a thin echo which moved rapidly forwards to and backwards from a midsystolic position, close to or impinging on the interventricular septum (Fig. 1, echo 3): it was not recorded in early or end-systole and had no demonstrable continuity with the mitral anterior cusp echo. These two echoes thus formed the anterior (Fig. 1, echo 3 ) and posterior (Fig. 1, echo 1) edges of the systolic complex, the third component of which was a series of echoes (Fig. 1, echo 2) with characteristics similar to those of the myocardium of the left ven- tricular posterior wall (Fig. 1). They were recorded between the anterior and posterior echoes in midsystole and were displayed as a narrow band in front of the posterior echo in early and late systole. Their continuity with the echoes of the left ventricular wall in the region of the posterior papillary muscle was apparent when the transducer was directed downwards (Fig. 2).

\section{Multiple element scans}

Echo images of the aortic root, the anterior leaflet of the mitral valve, and the hypertrophied septum were recorded in both phases of the cardiac cycle; echoes from the posterior leaflet and from the posterior walls of the left atrium and left ventricle were less clearly defined (Fig. 3 to 6 ).

Diastole The mitral anterior leaflet moved rapidly forwards in diastole to a position where its free margin and an adjacent portion of the cusp impinged on the interventricular septum and where its tip lay in a vertical plane approximately mid-way between the anterior and posterior walls of the aortic root (Fig. 3). During this opening movement, the entire anterior cusp was orientated parallel with or convex to the interventricular septum (Fig. 4). At end-diastole, the anterior cusp moved rapidly backwards to a position where all of it, including the free margin, lay behind the vertical plane of the aortic root. The anterior and posterior mitral leaflets moved in opposite direstions throughout diastole.

Systole Both mitral leaflets moved towards a closed position at end-diastole but the valve remained open. In early systole, though the anterior leaflet lay behind the vertical plane of the aortic 


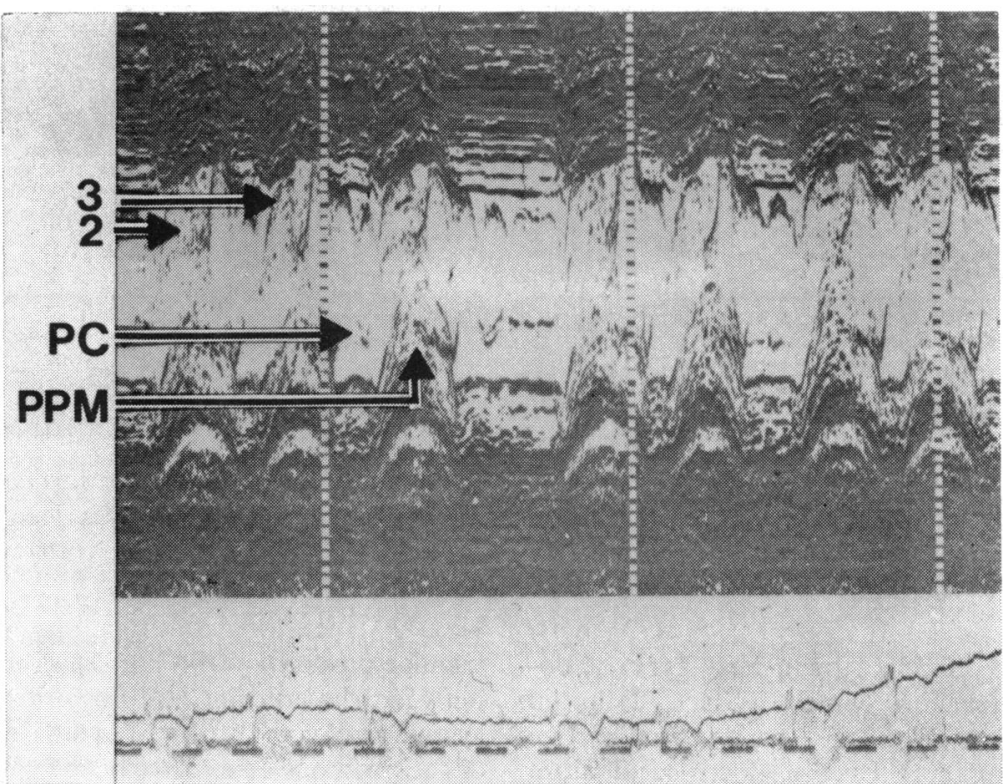

FIG. 2 Single dimensional echocardiogram with simultaneous electro- and phonocardiograms from the same patient as Fig. 1. The ultrasonic beam has been directed downwards to the region of the posterior papillary muscle. The echoes forming the systolic complex (see text) are numbered: continuity between echo 2 and the papillary muscle echoes is apparent in the fourth cardiac cycle. In this and subsequent figures : $P C=$ mitral posterior cusp, $P P M=$ posterior papillary muscle.

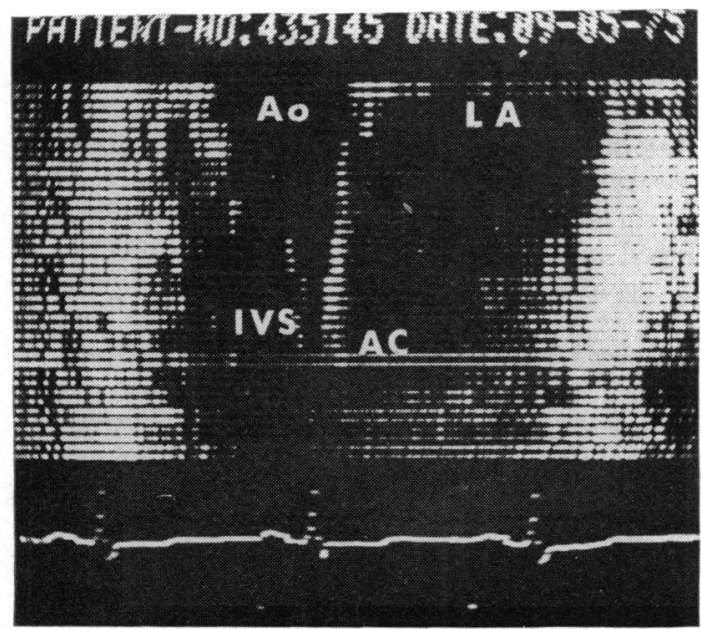

FIG. 3 Hypertrophic cardiomyopathy with obstruction: multidimensional echocardiogram in middiastole. The tip of the mitral anterior leaflet impinges on the in.erventricular septum. In this and subsequent figures: $A o=$ aortic root, $L A=$ left atrium. root and both leaflets presented a convex configuration towards the left atrium, their free margins were not in apposition (Fig. 5). Forward motion of both mitral cusps occurred in mid-systole: movement of the anterior cusp was maximal at, and close to, its free margin, and was slower and of smaller amplitude than the diastolic opening movement. Thus, while the remainder of the anterior leaflet maintained a more posterior position and a convex configuration towards the left atrium, its tip moved forwards into line with the posterior wall of the aorta and did not impinge on the septum (Fig. 6). At end-systole, the anterior cusp moved backwards for a short distance, but did not return to its more posterior end-diastolic position: details of posterior cusp motion were not defined.

Echoes which were not recorded in diastole intervened between the septal and anterior cusp echoes in systole (Fig. 5 and 6). The structure from which they originated moved upwards in the long axis of the ventricle in early systole to a position well above the tip of the anterior cusp. Its lower portion was closely related to the interventricular septum and, in mid-systole, to the tip of the mitral anterior 

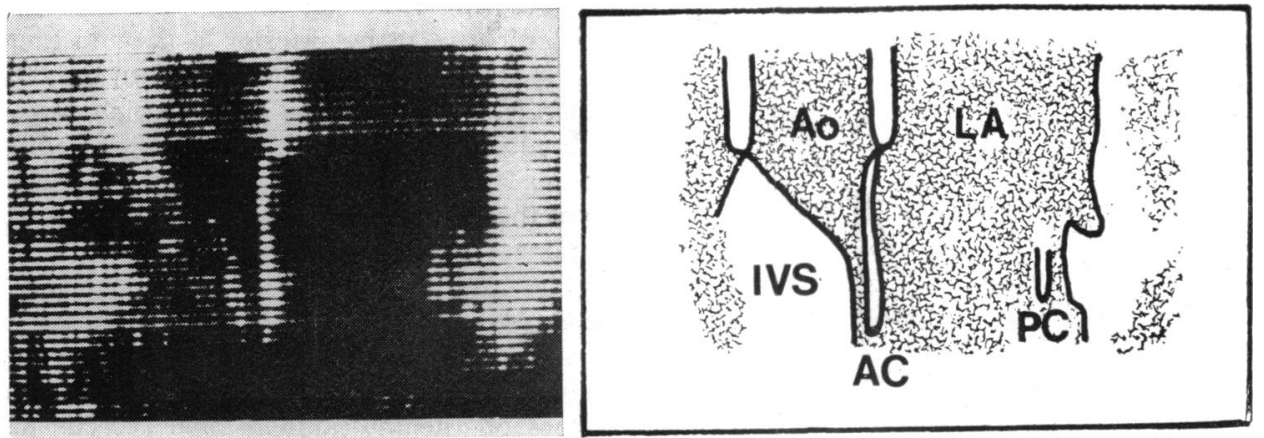

FI G. 4 Multidimensional echocardiogram in late diastole from the same patient as Fig. 3

The mitral anterior leaflet is closely related to, but not in contact with, the hypertrophied septum.

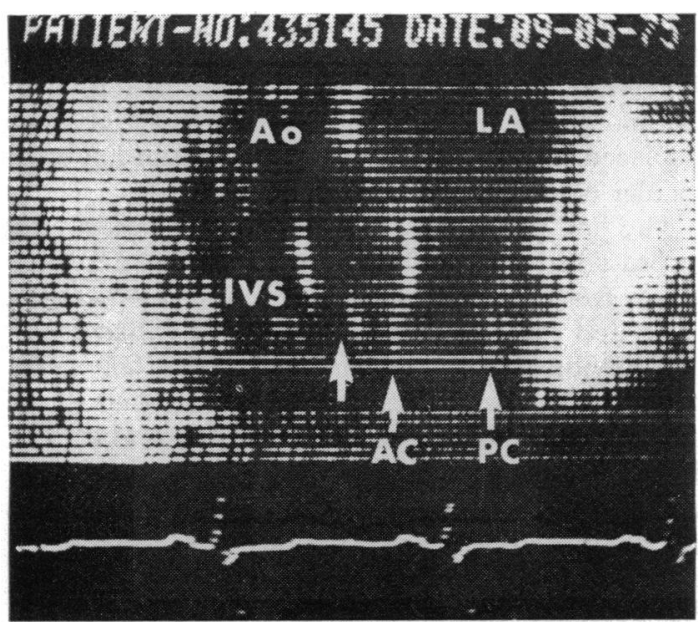

FIG. 5 Multidimensional echocardiogram in early systole from the same patient as Fig. 3. The mitral leaflets are not in apposition. The structure (arrowed) which intervenes between the anterior leaflet and the septum has been identified (see text) as the posterior papillary muscle. leaflet (Fig. 6). Forward motion of the anterior leaflet appeared to be halted in mid-systole by contact with this structure.

\section{Discussion}

The systolic abnormality of the conventional mitral echogram in hypertrophic cardiomyopathy with obstruction has been shown here to be an echo complex (Fig. 1). Previous workers (Shah et al., 1969; Pridie and Oakley, 1970; Henry et al., 1973) were thus wrong to ascribe it solely to systolic anterior motion of the mitral anterior leaflet. Its components have not been described before, though they can be seen in published tracings (Bolton et al., 1974; Tajik and Giuliani, 1974; Henry et al., 1975). For the reasons which follow, it has been concluded that they arise from the mitral anterior leaflet, the posterior papillary muscle, and the chordae tendineae (Fig. 1, echoes 1, 2, and 3, respectively).

Multiple element scanning has shown that the mitral anterior leaflet does not impinge on the inter-
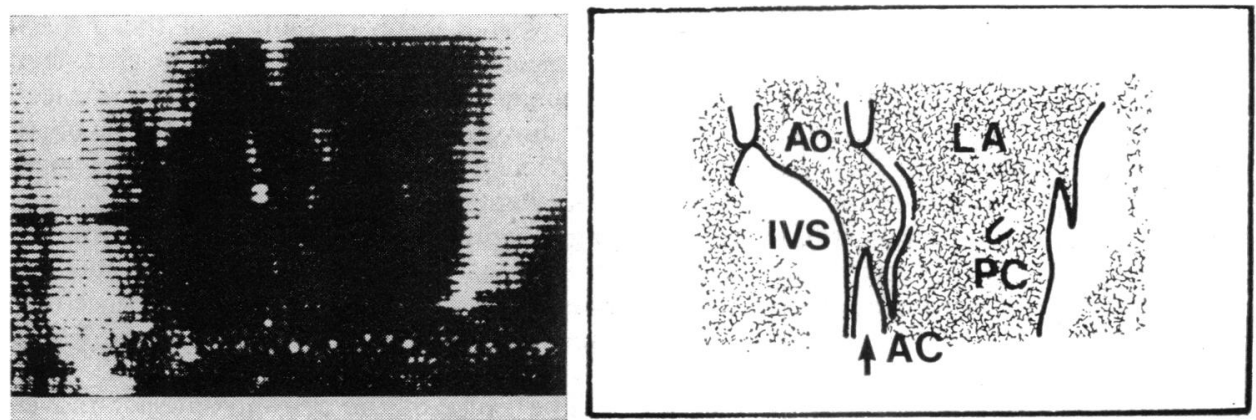

FIG. 6 Multidimensional echocardiogram in mid-systole from the same patient as Fig. 3. The structure (arrowed) on which the anterior leaflet impinges has been identified (see text) as the posterior papillary muscle. 


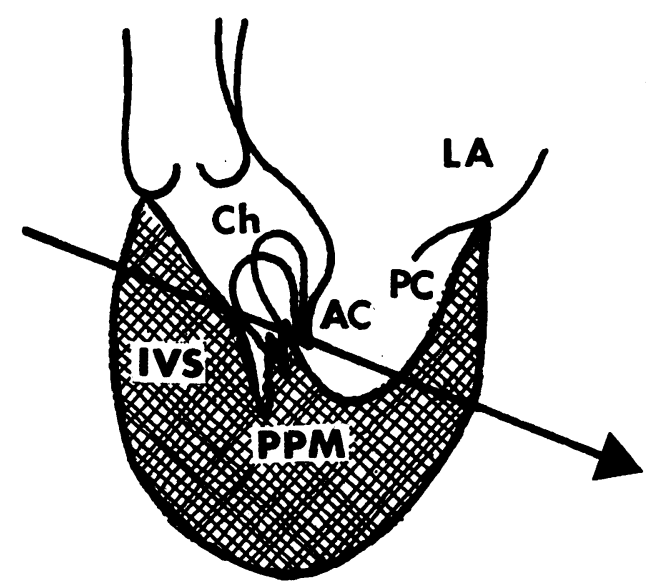

FIG. 7 Hypertrophic cardiomyopathy with obstruction: a diagram of the left heart in mid-systole. $\mathrm{Ch}=$ chordae tendineae. It is suggested that the posterior papillary muscle and the chordae tendineae are displaced upwards and forwards. An ultrasonic beam (arrow) directed to the tip of the anterior leaflet thus passes in sequence through the interventricular septum, the chordae, the posterior papillary muscle, the mitral anterior leaflet, and the left ventricular posterior wall.

ventricular septum in systole (Fig. 6) and that while systolic anterior movement of the leaflet does occur it is smaller and slower than its diastolic counterpart. Thus, the posterior element of the complex (Fig. 1, echo 1) is identified as the anterior leaflet echogram by its position, amplitude, and velocity as well as by its continuity with the anterior cusp echoes at end-diastole and end-systole. It follows that the two remaining elements of the complex arise from a structure or structures interposed between the septum and the anterior leaflet in systole. The only structures which could intervene in this position are the posterior papillary muscle and its chordae tendineae; echo images of all the other left heart structures accessible to ultrasound are recorded elsewhere on the systolic multiple element scans (Fig. 5 and 6). It has been concluded from their configuration that the echoes displayed in two dimensions between the anterior leaflet and the septum (Fig. 6) arise from the posterior papillary muscle: Henry et al. (1975) attributed similar though less anteriorly positioned echoes to papillary muscle, but did not specifically identify the posterior papillary muscle. Though it appears from the multidimensional records that the posterior papillary muscle is the sole structure interposed between the anterior leaflet and the septum, the single element scans suggest that the chordae also intervene. Thus, the differing characteristics of the two echoes recorded between the anterior leaflet and the septum in Fig. 1 are in keeping with their origins from two separate structures, only one of which (Fig. 1, echo 2 ) is muscular. It has, therefore, been deduced that echoes 2 and 3 of the systolic complex (Fig. 1) arise from the posterior papillary muscle and the chordae tendineae, respectively.

The interrelations of the echoes in Fig. 1 can be explained if, in systole, the septum and the components of the mitral apparatus are interrelated as suggested in Fig. 7. The failure to identify chordal echoes on the multidimensional records may well reflect the poor resolution of the multiple element ultrasound system. Henry et al. (1975), using a sector-scanner, achieved better echo definition: when their two-dimensional records are reviewed in the light of the present study, it is evident that the echoes which they have attributed to the tip of the anterior leaflet, could instead be from chordae displaced upwards and forwards into the left ventricular outflow tract, as in Fig. 7.

The findings of this investigation are not in total accord with the conclusions on mitral cusp motion drawn from angiographic (Simon, 1968; Reis et al., 1974) and previous echocardiographic (Shah et al., 1969; Pridie and Oakley, 1970; Henry et al., 1975) studies. Thus, while systolic anterior motion of the anterior leaflet is confirmed, systolic contact between the leaflet and the interventricular septum is not (Fig. 5 and 6). Though the concept of left ventricular outflow obstruction by the anterior leaflet is upheld, contact between the tip of the leaflet and the posterior papillary muscle, rather than the septum, appears to be responsible (Fig. 6). The failure of leaflet apposition in early systole, shown here (Fig. 5), has been suspected angiographically (Simon, 1968): it follows that the valve does not reopen in systole, as has been suggested (Pridie and Oakley, 1970), and also that the mitral regurgitation of hypertrophic cardiomyopathy may be pansystolic. Anterior motion of the posterior cusp was seen in systole, but it is clear that, contrary to Simon's (1968) hypothesis, the posterior leaflet does not move with the anterior leaflet to obstruct left ventricular outflow (Fig. 6). Anterosuperior displacement of both papillary muscles has been shown angiographically (Reis et al., 1974), but the close relation of the posterior papillary muscle to the interventricular septum and its displacement above the free margins of the mitral leaflets (Fig. 5 and 6) have not been emphasized before.

The cause of the systolic anterior movement of the mitral anterior cusp has been the subject of speculation (Simon, 1968; Shah et al., 1969; Pridie and Oakley, 1970; Reis et al., 1974; Henry 
et al., 1975). The theory that it is the result of pull by abnormally directed papillary muscles has not been substantiated by this study. Thus, the posterior papillary muscle is displaced above and in front of the mitral leaflets (Fig. 5): from this position, because the apices of the two structures are not aligned, it is not mechanically feasible for the papillary muscle to exert traction on the leaflets. Further, if papillary muscle shcrtening, which was not shown, does occur in hypertrophic cardiomyopathy, posterior papillary muscle pull will be directed along the ventricular long axis and will tend to pull the leaflets downwards rather than forwards. Because it precedes it (Fig. 5), displacement of the papillary muscle has also been discounted as the direct cause of systolic anterior motion of the leaflets.

The theory is put forward that, in the setting of the diminished left ventricular transverse dimension (Henry et al., 1975), anterosuperior displacement of the posterior papillary muscle produces chordal slackening, and that it is forwards and upwards displacement of the chordae by the blood flowing towards the aortic root during ventricular ejection that is responsible for systolic anterior motion of the leaflets. It is suggested that displacement of the chordae and anterior motion of the leaflets proceed until the anterior leaflet impinges on the posterior papillary muscle, producing left ventricular outflow obstruction. It is also postulated that failure of leaflet apposition in early systole is the result of papillary muscle displacement, with consequent loss of traction.

Posterior papillary muscle displacement is thus seen to be a fundamental abnormality which predisposes to systolic anterior motion of the leaflets and to left ventricular outflow obstruction, both of which can be regarded as functions of left ventricular ejection. It is of interest that, though their theories concerning mitral cusp motion are incompatible with the findings of this study, Henry et al. (1975) have concluded that hydrodynamic forces generated by left ventricular contraction are responsible for left ventricular outflow obstruction.

The lability of left ventricular outflow tract obstruction, its response to beta-adrenergic stimulation and blockade, and its disappearance as the systolic performance of the ventricle deteriorates (Goodwin and Oakley, 1972) are in keeping with the present hypothesis. Further support is lent to it by the fact that surgical relief of left ventricular outflow obstruction has been shown to depend on papillary muscle realignment (Reis et al., 1974).

It has been concluded from this study that the echocardiographic obstruction index developed by Henry et al. (1973) measures not left ventricular outflow obstruction but chordal displacement: the inaccuracies which have been observed (Rossen et al., 1974) are thus easily explained.

I wish to thank Dr. Robert Railton for his help with the technical aspects of the multidimensional study and Dr. Celia Oakley for her encouragement. The Echocardiovisor was supplied by the Scottish Home and Health Department for evaluation.

\section{References}

Bolton, M. R., King, J. F., Polumbo, R. A., Mason, D., Pugh, D. M., Reis, R. L., and Dunn, M. I. (1974). The effects of operation on the echocardiographic features of idiopathic hypertrophic subaortic stenosis. Circulation, 50, 897.

Goodwin, J. F., and Oakley, C. M. (1972). Editorial. The cardiomyopathies. British Heart fournal, 34, 545.

Henry, W. L., Clark, C. E., Glancy, D. L., and Epstein, S. E. (1973). Echocardiographic measurement of the left ventricular outflow gradient in idiopathic hypertrophic subaortic stenosis. New England fournal of Medicine, 288, 989.

Henry, W. L., Clark, C. E., Griffith, J. M., and Epstein, S. E. (1975). Mechanism of left ventricular outflow obstruction in patients with obstructive asymmetric septal hypertrophy (idiopathic hypertrophic aubaortic stenosis). American fournal of Cardiology, 35, 337.

Pridie, R. B., and Oakley, C. M. (1970). Mechanism of mitral regurgitation in hypertrophic obstructive cardiomyopathy. British Heart fournal, 32, 203.

Roelandt, J., Kloster, F. E., ten Cate, F. J., van Dorp, W. G., Honkoop, J., Bom, N., and Hugenholtz, P. G. (1974). Multidimensional echocardiography. An appraisal of its clinical usefulness. British Heart fournal, 36, 29.

Reis, R. L., Bolton, M. R., King, J. F., Pugh, D. M., Dunn, M. I., and Mason, D. T. (1974). Anterior-superior displacement of papillary muscles producing obstruction and mitral regurgitation in idiopathic hypertrophic subaortic stenosis. Circulation, 50, Suppl. II, 181.

Rossen, R. M., Goodman, D. J., Ingham, R. E., and Popp, R. L. (1974). Echocardiographic criteria in the diagnosis of idiopathic hypertrophic subaortic stenosis. Circulation, 50, 747.

Shah, P. M., Gramiak, R., and Kramer, D. H. (1969). Ultrasound localization of left ventricular outflow obstruction in hypertrophic obstructive cardiomyopathy. Circulation, 40, 3.

Simon, A. L. (1968). Angiographic diagnosis of idiopathic hypertrophic subaortic stenosis. Radiologic Clinics of North America, 6, 423.

Tajik, A. J., and Giuliani, E. R. (1974). Echocardiographic observations in idiopathic hypertrophic subaortic stenosis. Mayo Clinic Proceedings, 49, 89.

Requests for reprints to Dr. J. Christine Rodger, Division of General Medicine Unit B, Stobhill General Hospital, Glasgow G21 3UW. 